\title{
Corrigendum: Spatiotemporal Clustering of Repeated Super-Resolution Localizations via Linear Assignment Problem
}

\author{
David J. Schodt and Keith A. Lidke* \\ Department of Physics and Astronomy, University of New Mexico, Albuquerque, NM, United States
}

Keywords: fluorescence microscopy, super-resolution, image analysis, computational modeling, single molecule techniques

\section{A Corrigendum on}

Spatiotemporal Clustering of Repeated Super-Resolution Localizations via Linear Assignment Problem

by Schodt D. J and Lidke K. A (2021). Front. Bioinform. 1:724 325. doi: 10.3389/fbinf.2021.724325

In the original article, there was an error. Eq. (2) was written incorrectly. A correction has been made to MATERIALS AND METHODS, Estimating Local Emitter Densities and Kinetic Rates, Paragraph Number 2:

The expected cumulative number of localizations observed by frame $f$ is given by

\section{OPEN ACCESS}

Edited and reviewed by: Thomas Pengo,

University of Minnesota Twin Cities, United States

*Correspondence: Keith A. Lidke klidke@unm.edu

Specialty section:

This article was submitted to Computational Biolmaging, a section of the journal

Frontiers in Bioinformatics

Received: 15 December 2021 Accepted: 21 December 2021 Published: 25 January 2022

Citation: Schodt DJ and Lidke KA (2022) Corrigendum: Spatiotemporal Clustering of Repeated SuperResolution Localizations via Linear Assignment Problem.

Front. Bioinform. 1:836606. doi: 10.3389/fbinf.2021.836606

$$
\left\langle n_{\text {cumulative }}\right\rangle(f) \approx N_{\text {emitters }}\left(1-p_{\text {miss }}\right) \tau\left\{\frac{1-\exp \left[-\lambda_{1}(f-1)\right]}{\lambda_{1}}-\frac{1-\exp \left[-\lambda_{2}(f-1)\right]}{\lambda_{2}}\right\}
$$

In the original article, there was an error. An unlabeled equation was written incorrectly. Additionally, a line of text in the associated paragraph was written incorrectly. A correction has been made to MATERIALS AND METHODS, Estimating Local Emitter Densities and Kinetic Rates, Paragraph Number 4:

The local pre-cluster density corresponding to each pre-cluster is estimated by finding the $k$ (chosen to be two in this study) nearest pre-clusters and then computing $\rho_{c}=(k+1) / \pi r_{k}^{2}$ where $r_{k}$ is the distance to the $k$ th nearest pre-cluster. The underlying local emitter density present at the beginning of the experiment is then estimated for each pre-cluster as

$$
\hat{\rho}_{0, \text { local }}=\rho_{c} \frac{1}{\hat{k}_{\text {off }} \hat{\tau}} \frac{1}{1-\hat{p}_{\text {miss }}}\left\{\frac{1-\exp \left[-\hat{\lambda}_{1}\left(f_{\text {end }}-1\right)\right]}{\hat{\lambda}_{1}}-\frac{1-\exp \left[-\hat{\lambda}_{2}\left(f_{\text {end }}-1\right)\right]}{\hat{\lambda}_{2}}\right\}^{-1}
$$

where $f_{\text {end }}$ is the last frame containing localizations in the experiment.

The authors apologize for this error and state that this does not change the scientific conclusion of the article in any way. The original article has been updated.

\footnotetext{
Publisher's Note: All claims expressed in this article are solely those of the authors and do not necessarily represent those of their affiliated organizations, or those of the publisher, the editors and the reviewers. Any product that may be evaluated in this article, or claim that may be made by its manufacturer, is not guaranteed or endorsed by the publisher.
}

Copyright (c) 2022 Schodt and Lidke. This is an open-access article distributed under the terms of the Creative Commons Attribution License (CC BY). The use, distribution or reproduction in other forums is permitted, provided the original author $(s)$ and the copyright owner(s) are credited and that the original publication in this journal is cited, in accordance with accepted academic practice. No use, distribution or reproduction is permitted which does not comply with these terms. 